\title{
An MDA Method for Service Modeling by Formalizing REA and Open-edi Business Frameworks with SBVR
}

\author{
Jelena Zdravkovic, Iyad Zikra, and Tharaka Ilayperuma \\ Department of Computer and Systems Sciences \\ Stockholm University and Royal Institute of Technology \\ Forum 100, SE-164 40 Kista, Sweden \\ \{jelenaz, iyad,si-tsi\}@dsv.su.se
}

\begin{abstract}
Business frameworks offer great opportunities of communication between people for working on the enterprise system engineering processes, as well as for eliciting services that the enterprise can offer in collaboration contexts. However, these kinds of frameworks, such as Resource-Event-Agent and Open-edi, recently unified in Open-edi Business Ontology (OeBTO), lack formal representations. This fact considerably limits their use in system development, particularly in model-driven development methods where the efficiency of transformations is of great importance. In this paper we suggest a formalization of OeBTO using OMG's standard Semantics of Business Vocabulary and Business Rules (SBVR), as a method for creating a service-centric business model. This makes it possible to provide the necessary formal logic foundation to allow automatic processing of the business model and its transformation to a system-level service model. An example from the bank loan business sector is used to argument the application of the method.
\end{abstract}

Keywords: business model, business collaboration, service engineering, modeldriven development, REA, Open-edi, OeBTO, MDA, SBVR.

\section{Introduction}

Model Driven Architecture (MDA), as a formalization of Model Driven Development (MDD) approach, promotes a method for system development relying on the model transformation paradigm. MDA prescribes modeling of the business-level information as a Computational Independent Model (CIM), which is further transformed to a system-centric form called Platform Independent Model (PIM), and at the end to a Platform Specific Model (PSM) that adds the technology details needed for implementation on a specific software platform [1].

In the service-oriented business sector, capturing the consumer needs for economic resources plays an essential role in the elicitation of the services that will deliver these values, therein seizing a desirable competitive distinction. In that context, business models offer considerable advantages compared to process models - they can capture a high-level description of a whole business in a single and easily-understandable view. Business ontologies, such as Resource-Event-Agent, REA [2], facilitate modeling of actors involved in a business scenario and explain their relationships, 
formulating them in terms of economic values (i.e. resources) exchanged between the actors. Another important aspect concerns elicitation of explorative business service portfolios by spanning the whole business transaction lifecycle, which, according to the International Organization for Standardization (ISO) Open-edi initiative [3] involves planning, identification, negotiation, actualization, and post-actualization.

Recently, the ISO has focused on integrating REA and the Open-edi frameworks to create Open-edi Business Transaction Ontology (OeBTO), to specify the concepts and relationships involved in collaborative business environments. OeBTO captures the economic commitments realized by economic and business events performed by the partners, along the collaboration lifecycle in the Open-edi sense.

Following the previously outlined needs of service engineering, and MDA, in this study we consider the use of OeBTO to define a service-centric business model (i.e. CIM) and a method for its creation. To strengthen the formalism of OeBTO and thereby create an unambiguous and processable CIM, we consider the use of the OMG's Semantics of Business Vocabulary and Business Rules [4]. SBVR is an approach which allows specifying business in terms of a vocabulary and rules in a business-friendly language, while being formal enough to be readable by systems.

Being rooted in the use of MDA and two well-established business frameworks; REA and Open-edi formalized by SBVR, we believe that the method we propose for creating CIM forms a solid basis to be efficiently transformed to a SOA-aligned system model and further to Web services.

The rest of the paper is organized as follows. Section 2 gives the overviews on the used business frameworks, and SBVR. In Section 3 we present our method for identification and modeling of business services. In Section 4, we refer to the work related to ours, and conclude the paper.

\section{Background}

In this section, we briefly describe REA and Open-edi business frameworks and their integration in Open-edi Transaction Ontology (OeBTO); then we give an overview of the Semantics of Business Vocabulary and Business Rules (SBVR) standard.

\section{Integration of REA and Open-edi Business Frameworks in OeBTO}

The core concepts in the Resource-Event-Agent (REA) framework are resource, event, and agent [2]. It is assumed that every business activity can be described as an event where two agents exchange economic values, i.e. resources. Economic resources may be classified as goods, rights or services. To acquire a resource, an agent (i.e. actor) has to give up some other resource (economic duality). In the study [5], the REA framework has been extended to capture additional granularity levels of business activities of enterprises. The resulting framework has integrated three vertical layers: Value Chain, Business Process and Business Event:

Open-edi Business Transaction Ontology (OeBTO) extends the REA ontology with the concepts aimed to facilitate the modeling of business collaborations defined in the ISO Open-edi initiative [6]. According to Open-edi, business collaborations span five phases: planning, identification, negotiation, actualization and postactualization. In the planning phase, the customer and the provider are engaged in 
activities to identify the actions needed for selling or purchasing goods and services. The identification phase involves the activities needed to exchange information among providers and potential customers regarding selling or purchasing goods and services. During the negotiation phase, contract terms are proposed and completed. The actualization phase includes all the activities necessary for exchanging goods and services between involved actors as agreed during negotiations. The postactualization phase encompasses the activities and associated exchanges between involved actors after the major resources are provided.

\section{SBVR}

Recently, the Object Management Group (OMG) has adopted the Semantics of Business Vocabulary and Business Rules (SBVR) as a standard for capturing business vocabularies and rules [4]. The term "business" in SBVR is used in a general sense, referring not only to activities that imply an exchange of goods or services for money, but also to other types of activities where rules need to be defined and documented, such as education, health care, or law [7].

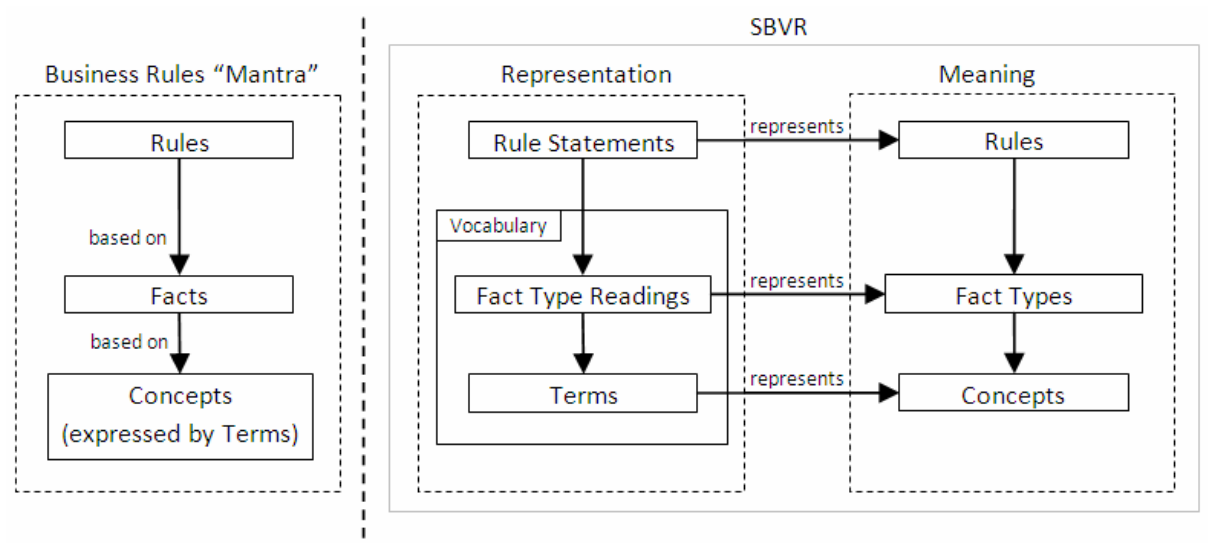

Fig. 1. Meaning, representation, and the support of business rules in SBVR

The basic idea around which SBVR is developed is that business rules are built on top of facts, which in turn are built on top of terms that represent concepts. Furthermore, SBVR acknowledges the difference between meaning and the representation used to convey that meaning. Figure 1 above shows how SBVR realizes the business rules and also emphasizes the independence of meaning and its representation.

\section{Creating Service-Centric SBVR-Based Business Model}

In this study we utilize the MDA method to model services. In that effort, we consider REA and Open-edi (i.e. OeBTO) as an established conceptual basis for the business collaboration context. To obtain a service-centric CIM, we propose the following:

- A classification of the notion of Business Transaction in OeBTO to enable CIM to describe different value configurations. 
- An extension to the original OeBTO to capture service-related notions, such as structure, behavior or policies.

- A (re)formulation of OeBTO using SBVR, to increase the formalism of CIM and thereby facilitate transformations to the PIM level.

\section{Method for Creating a Three-layered Business Model}

According to the REA framework, the method for describing the business of an enterprise comprises the decomposition of business activities along three granularity layers: value chain, business processes and business events (Figure 2). In the following, we will outline a method for creating each of the layers, where the concepts on each of the layers are defined using SBVR-based OeBTO.

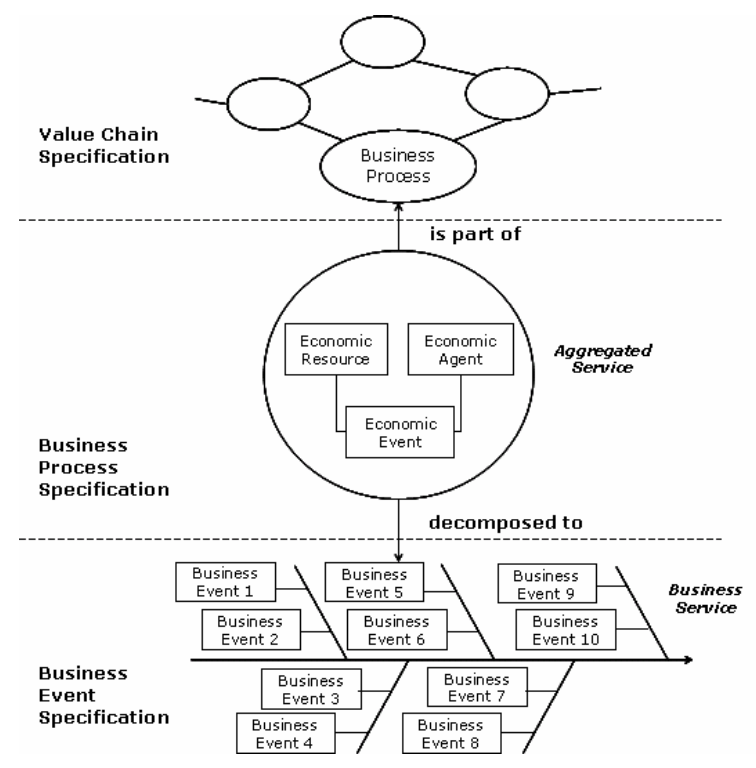

Fig. 2. The three-layered REA business framework

\section{Step 1: Value Chain Specification}

In this step the business (i.e. value-adding) processes in the highest layer of the REA framework are identified, using a service-aware value configuration, such as the classification that includes traditional value chain, value shop, and value network [8].

Step 2: Business Process Specification

Moving to the middle layer of the REA framework, each identified business process (transaction, in OeBTO) is explored to find the partners involved in it, as well as the economic resources being exchanged. Each economic exchange gives rise to an $a g$ gregated service, which will be further expanded on the next layer of the framework, to discover the actual business services that will realize the delivery of the economic exchange. 


\section{Step 3: Business Event Specification}

At the bottom layer of the REA framework, the economic events of the economic exchange are expanded over the five Open-edi business transaction phases. This is intended to discover the candidate business services and business events. According to OeBTO, the business event is used to represent the business activities elicited for every business transaction phase at the third layer. In order to capture the services that compose the aggregated service and at the same time aggregate related business events, we introduce the business service element. A business service is a standalone service that can be reused as part of other aggregated services to provide other economic exchanges. In Figure 3, a small excerpt of the formalization of OeBTO using SBVR is illustrated. For the full specification, the reader is referred to [9]

\section{aggregated service defines economic exchange}

Definition: the aggregated service describes the aggregation of services which will realize and support the delivery of the economic exchange.

\section{business transaction phase includes business service}

Necessity: business transaction phase includes at least one business service

Fig. 3. An excerpt of the formalization of OeBTO using SBVR

From the MDA perspective, the obtained service-aware OeBTO model is used as the input for creating a system model, i.e. Platform Independent Model (PIM). Since SBVR is completely grounded in formal logic, it gives the added benefit of automatic model processing, i.e.:

- Making it possible for tools to ensure the integrity of OeBTO models.

- Ensuring the integrity of transformations that produce PIM models based on the OeBTO model.

\section{Related Work and Conclusion}

In this study, we have applied the MDA method to design business-services, which may be further transformed to system services and implemented using Web services.

Lately, research in both academic and industrial communities have implied that when designing service-oriented software solutions, the starting point should be the business models of enterprises [10], [11], [12] and [13]. This fact, according to the referred studies, is shifting the focus of large scale e-service design to the context of economic resource transfers. Our method reported in [14] differs from those studies in the way that we set the focus on the analysis of business transactions relying on the OeBTO standardization effort, and expanding them along a number of collaboration phases to get a rich business service portfolio. The aim of this study has been to further improve the use of OeBTO for service modeling in the MDA method, by formalizing it with SBVR, and to use SBVR to guide transformations toward PIM.

The major strength of the proposed method is the use of REA and Open-edi frameworks formalized with SBVR for modeling CIM; in that way we have obtained a method which facilitate creating a declarative-type CIM, unambiguous and 
processable, i.e. with the capability to be further transformed with a high extent of automation to system-centric service model (PIM).

\section{References}

1. Kleppe, A., Warmer, J., Bast, W.: MDA Explained. Addison-Wesley Professional, Reading (2003)

2. McCarthy, W.E.: The REA Accounting Model: A Generalized Framework for Accounting Systems in a Shared Data Environment. The Accounting Review (1982)

3. ISO/IEC: Operational aspects of Open-edi implementation. ISO Standard 15944-1 (2002)

4. The Object Management Group (OMG): Semantic of Business Vocabulary and Business Rules (SBVR), v1.0. OMG standard, http: / /www. omg.org/spec/SBVR/1.0/PDF

5. Geerts, G., McCarthy, W.E.: An Ontological Analysis of the Primitives of the ExtendedREA Enterprise Information Architecture. The International Journal of Accounting Information Systems 3, 1-16 (2002)

6. ISO/IEC: Business transaction scenarios - Accounting and economic ontology. ISO Standard 15944-4 (2007)

7. Linehan, M.H.: SBVR Use Cases. In: Bassiliades, N., Governatori, G., Paschke, A. (eds.) RuleML 2008. LNCS, vol. 5321, pp. 182-196. Springer, Heidelberg (2008)

8. Stabell, C.B., Fjeldstad, O.D.: Configuring value for competitive advantage: on chains, shops, and networks. Strategic Management Journal 19, 413-437 (1998)

9. Zikra, I.: SBVR-based Service-extended OeBTO Meta Model. Technical Report, http://people.dsv.su.se/ iyad/public/SBVR-Based_OeBTO_Meta_ Model.pdf

10. Baida, Z., Gordijn, J., Saele, H., Akkermans, H., Morch, A.: An Ontological Approach for Eliciting and Understanding Needs in e-Services. In: Pastor, Ó., Falcão e Cunha, J. (eds.) CAiSE 2005. LNCS, vol. 3520, pp. 400-414. Springer, Heidelberg (2005)

11. Cherbakov, L., Galambos, G., Harishankar, R., Kalyana, S., Rackham, G.: Impact of Service Orientation at the Business Level. IBM Systems Journal 44(4), 653-668 (2005)

12. Anderssson, B., Johannesson, P., Zdravkovic, J.: Aligning Goals and Services through Goal and Business Modeling. The International Journal of Information Systems and eBusiness Management (ISEB), Special Issue on Design and Management of Business Models and Processes in Services Science 7, 143-169 (2007)

13. Gordijn, J., van Eck, P., Wieringa, R.: Requirements Engineering Techniques for eServices. In: Georgakopoulos, D., Papazoglou, M.P. (eds.) Service-Oriented Computing: Cooperative Information Systems Series, pp. 331-352. The MIT Press, Cambridge (2009)

14. Zdravkovic, J., Ilayperuma, T.: A Model-driven Approach for Designing E-Services Using Business Ontological Frameworks. In: Proceedings of 14th IEEE International EDOC Conference, pp. 121-130. IEEE Computer Society, Los Alamitos (2010) 